\title{
What about using sniffin' sticks 12 items test to screen post-COVID-19 olfactory disorders?
}

\author{
Clair Vandersteen ${ }^{1,2}$ (D) Magali Payne ${ }^{2,3} \cdot$ Louise-Émilie Dumas $^{2,5} \cdot$ Alexandra Plonka $^{2,4} \cdot$ Grégoire D'Andréa $^{1}$. \\ David Chirio $^{6} \cdot$ Élisa Demonchy $^{6} \cdot$ Karine Risso $^{6} \cdot$ Philippe Robert $^{2} \cdot$ Xavier Fernandez $^{7}$. Florence Askenazy-Gittard ${ }^{2}$. \\ Charles Savoldelli ${ }^{1} \cdot$ Nicolas Guevara $^{1} \cdot$ Laurent Castillo $^{1} \cdot$ Valeria Manera $^{2,4} \cdot$ Auriane Gros ${ }^{2,3}$
}

Received: 4 October 2021 / Accepted: 18 October 2021 / Published online: 30 October 2021

(c) The Author(s), under exclusive licence to Springer-Verlag GmbH Germany, part of Springer Nature 2021

\begin{abstract}
Background Post-COVID-19 Olfactory impairment has a negative impact on quality of life. The Sniffin Sticks test 12 items (SST-12) can be used in quick olfactory disorders screening. Its evaluation in a post-covid-19 situation was the main objective of this work.

Methods All patient impaired with a post-COVID olfactory loss were included while consulting to the ENT department. The clinical examination included an olfaction recovery self-assessment (VAS), a nasofibroscopy, a quality of life (QoL) assessment, the complete Sniffin' Sticks Test (SST), and the SST-12.

Results Among the 54 patients included, 92\% $(n=50)$ were correctly screened as olfactory impaired by SST-12. We report excellent correlations between SST-12 and SST ( $\left.r \mathrm{rho}_{(52)}=0.98, p<0.001\right)$, $\mathrm{QoL}\left(\mathrm{rho}_{(52)}=0.33 p=0.016\right)$, or VAS $\left(\right.$ rho $\left._{(52)}=0.49, p<0.001\right)$ assessments.

Conclusions SST-12 is a quick and reliable tool to screen large-scale population of post-COVID-19 olfactory impaired patients and could be used in a general daily clinical practice.
\end{abstract}

Keywords Olfactory dysfunction $\cdot$ Screening $\cdot$ Sniffin sticks test 12 items $\cdot$ Covid- $19 \cdot$ Olfaction

Clair Vandersteen

vandersteen.c@chu-nice.fr

1 Institut Universitaire de La Face Et du Cou, 31 Avenue de Valombrose, 06100, Centre Hospitalier Universitaire, Université Côte d'Azur, Nice, Alpes-Maritimes, France

2 Université Côte d'Azur, Centre Hospitalier Universitaire de Nice, Laboratoire CoBTeK, Service Clinique Gériatrique du Cerveau Et du Mouvement, Nice, France

3 Université Côte d'Azur, Département d'Orthophonie de Nice, UFR Medecine, Nice, France

4 Institut NeuroMod, INRIA Centre de Recherche Sophia Antipolis, Université Côte d'Azur, 2004 Route des Lucioles, 06902 Sophia Antipolis, Alpes-Maritimes, France

5 Hôpitaux Pédiatriques de Nice CHU-LENVAL, Centre Hospitalier Universitaire, Université Côte d'Azur, 57 Avenue de la Californie, 06200 Nice, Alpes-Maritimes, France

6 Département de Médecine Infectiologique, Hôpital de L'archet, Centre Hospitalier Universitaire, Université Côte d'Azur, 151 route de Saint-Antoine, 06200 Nice, Alpes-Maritimes, France

7 Institut de Chimie de Nice, Université Côte d'Azur, Nice, Alpes-Maritimes, France

\section{Introduction}

The onset of a sudden partial (hyposmia) or total (anosmia) loss of smell is now recognized as highly predictive of SARS-COV-2 infection [1]. A long-lasting anosmia can lead to a quality of life [2] impairment, bad diet habits [3], changes in social relations $[4,5]$ psychiatric disorders, such as depression [6], anxiety, or anorexia [7] and its nutritional consequences [8], and finally cognitive impairment [4]. Thus, olfactory disorders have to be diagnosed and managed especially as 12 [9] months after the COVID-19 infection, $30 \%$ of patients retain an olfactory complaint and require attention.

Although there are different ways to assess a patient's ortho and retro-olfaction [10,11], only 50\% of ENTs assess the olfactory disorders on an anamnesis, and $10 \%$ assess through psychophysical tests [12]. Olfaction is most often evaluated by subjective self/hetero questionnaires with a significant variability of the results and a probable underestimation [12], given the poorer olfactory perception before 20 years and after $50[13,14]$. Complete psychophysical 
olfactory tests, with assessment of odor threshold, odor discrimination, and odor identification, are the gold standard [12] and allow to specify the olfactory disorder [15]. The most used in Europe is the Sniffin' sticks test ${ }^{\circledR}$ (SST) [12, 16] that includes an odor Threshold detection (T), an odor Discrimination (D), and an odor Identification (I) tests. However, these psychophysical tests are expensive and take a long time (between 30 and $60 \mathrm{~min}$ )) [10, 11], thus making their daily clinical use difficult. It therefore seems important to look for other olfactory tests that are faster $(\leq 5 \mathrm{~min})$ and accessible to specialists, but also to general practitioners.

The Sniffin' Sticks Test 12 items (SST-12) is an olfactory screening test in the form of a 4-min identification test allowing, according to its authors, to detect anosmia and hyposmia with comparable measurement reliability other similar scent screening tests [17-19]. It can also be used laterally (one nostril tested independently of the other).

Seeing that it has been demonstrated that identification impairment is predominant in post-COVIDs $[2,20]$, the objective of this study was to assess the potential value of SST-12 in screening and characterizing a persistent postCOVID-19 olfactory disorder in a daily practice.

\section{Material and methods}

\section{Population}

This study was approved by the institutional review board of the Nice University Hospital (CNIL number: 412) and registered with a ClinicalTrials.gov number (ID: NCT04799977). Since March 2020, we prospectively enrolled patients at ENT division of Nice University Hospital from March 2020 to May 2021. All were contaminated by COVID-19 with persistent olfactory disorders more than 6 weeks (2 to 14 months). Patients where mainly self-referred or referred by general practitioners or colleagues. Patients had either a RT-PCR-proven SARS-CoV-2 diagnosis or a CT-proven SARS-CoV-2 diagnosis secondarily confirmed by serology. We retrospectively extracted patients' demographic data and clinical features, including nasofibroscopy, subjective taste impairment, visual analog scale (VAS) for the subjective assessment of olfactory recovery (ranging from 0 to 100\%), diet habits modifications (over consumption of salt and sugar), and olfactory quality of life. Total and subdomains SST [21-23] score results were systematically assessed.

\section{Sniffin' sticks test 12 items}

Olfaction diseases SST-12 test has been validated in 2001 by Hummel et al. [19]. This 4-min screening psychophysical test is an odor identification test based on 12 from the
16 odors being sniffed during the identification subdomain part of the original SST.

The original SST identification odors set include peppermint, orange, fish, leather, rose, cloves, coffee, pineapple, licorice, anise, lemon, banana, cinnamon, apple, turpentine, and garlic. During the identification SST test, subjects were blindfolded. Sixteen odorant sticks were presented once, separated by an interval of at least $20 \mathrm{~s}$ to prevent olfactory desensitization. Each stick presentation was accompanied by a written list containing the correct odorant and 3 semantic distractors. Retrospectively, results from all odors set but apple, turpentine, garlic and anise were summed up to the SST-12 global score, as previously described [19]. We defined a normosmia (SST-12 $\geq 11$ ), an hyposmia $(10>$ SST-12 $>6)$, or an anosmia (SST-12 $\leq 6)$ based on normative values assessed from more than 1200 patients assessed with SST and olfactive evoked potential for anosmic and hyposmic ones [19]. Apple, turpentine, and garlic have been removed from the SST-12 because identified by less than 55\% of its normosmic validation cohort [19]. Anise was removed too because of being too similar to liquorice. With a reproducibility kappa coefficient of 0.77 , the diagnosis agreement can be considered as "good" (Altman, 1991). Although olfactory abilities decreased at extreme ages, SST12 can be used before the age of 10 and after the age of 80 .

\section{Olfactory quality of life}

The Questionnaire of Olfactory Disorders (QOD) is a widely validated tool related to olfaction quality of life evaluation [24]. Fifty-two items are reported in the original version regarding negative and positive social impacts of olfactory loss [25], but only negative statements subdomain of QOD(QOD-NS) [26] has been shown to be more consistent with SST results [27]. Moreover, being shorter than the original QOD, QOD-NS is more suitable for daily clinical practice $[28,29]$, increasing the response rate and reducing patient's mental burden while using it [28]. In order to improve QOD-NS, Mattos et al. [28] developed an even shorter version (Short-QOD-NS) with 7 most consistent questions related to social aspect $(n=3)$, eating $(n=2)$, anxiety $(n=1)$, and annoyance $(n=1)$ following an olfactory loss. Being now validated [30] in French [31], ShortQOD-NS was used this study allowing to evaluate olfactory quality of life with a score ranging from 0 to 21 (21 means there is no disorder).

\section{Statistical analysis}

Quantitative variables are presented as mean (SD) and qualitative variables as frequency and percentage. The degree of accordance between the SST and the SST-12 in patients' categorization was calculated using Cohen's Kappa coefficient. 
Sensitivity and specificity of the SST-12 compared to the SST in classifying patients as anosmic were also reported. To verify whether patients who increased their consumption of salt and sugar had lower SST, SST-12, and Short-QODNS scores compared to those who did not, we employed Mann-Whitney $U$ tests. Chi2 tests were employed to explore links between self-reported taste disorders and the presence of an increased salt and sugar consumption. To investigate correlations between subjective reports (VAS), odor identification disorders (based on the SST and SST-12), and QOD, we performed bivariate correlation analyses. As data were not normally distributed (as suggested by Kolmogorov-Smirnov test), non-parametric Spearman's correlations were employed. All tests were considered significant for alpha $<0.05$.

\section{Results}

\section{Demographic and clinical features}

We included fifty-four patients from the ENT department of Nice University Hospitals (CHU) complaining about olfactory loss, 5.4 \pm 3.1 months after a COVID-19 infection. Clinical features and demographic data are reported in Table 1. Forty-three of patients were male $(n=23)$, with a mean age of $39.9 \pm 13.9$ years. 17 patients $(31.5 \%)$ received a COVID-19 dedicated treatment, but no one did olfactory training before. All patients had a mild illness form.

\section{Retrospective olfactory and taste complains screening results}

Subjective, psychophysical, and quality of life tests results relatives to the loss of smell are reported in Table 2. On the day of consultation, patients reported to have recovered only $33.9 \pm 25.6 \%$ of their olfaction (ranging from 0 to $90 \%) .90 .7 \%$ of the patients $(n=49)$ reported taste disorders divided among retro-olfaction (food flavors) alone (64.8\%, $n=35)$, retro-olfaction associated to taste $(22.2 \%, n=12$; $16.7 \%$ concerning sweet and salty, $11.1 \%$ concerning sour and bitter), or taste alone $(1.8 \%, n=1$ concerning sweet and salty). $45.5 \%$ of patients ( 20 out of the 44 who responded to the question) reported that they increased their consummation of salt, and $20.5 \%$ ( 9 out of 44 ) that they increased their consummation of sugar. In terms of self-reported symptoms, $90.7 \%$ of the patients $(n=49)$ reported to suffer from anosmia, $7.4 \%(n=4)$ from hyposmia, $20.4 \%(n=11)$ from parosmia, and $24.1 \%(n=13)$ from phantosmia.

Categorization of patients based on the results of the olfactory tests is presented in Table 3. The global results (TDI) of the Sniffin' Sticks Test (SST) suggested that $24.1 \%(n=13)$ of the patients could be classified as
Table 1 Demographic and clinical characteristics

\begin{tabular}{lll}
\hline & $n$ & $\%$ \\
\hline Total & 54 & 100 \\
COVID-19 testing & & \\
Molecular PCR test & 46 & 85.2 \\
Chest CT & 11 & 20.4 \\
Serology (antibody test) & 16 & 29.6 \\
COVID-19 dedicated treatment & & \\
Oral corticosteroids & 6 & 11.1 \\
Nasal corticosteroids & 4 & 7.4 \\
Inhaled corticosteroids & 2 & 3.7 \\
Azithromycin alone & 7 & 13.0 \\
Hydroxychloroquine alone & 1 & 1.9 \\
Azithromycin + Hydroxychloroquine & 3 & 5.5 \\
Amoxicillin alone & 1 & 1.9 \\
Amoxicillin + Azithromycin & 2 & 3.7 \\
Others (vitamins, zinc) & 5 & 9.3 \\
Self-reported olfactory disorders & & \\
Hyposmia & 12 & 7.4 \\
Anosmia & 4 & 90.7 \\
Parosmia & 49 & 20.4 \\
Phantosmia & 11 & 24.1 \\
Taste disorders & 13 & 90.7 \\
Retro-olfaction alone & 49 & 64.8 \\
Retro-olfaction + taste & 35 & 22.2 \\
Taste alone & 12 & \\
\hline & & \\
& &
\end{tabular}

$C T$ computerized tomography, $P C R$ polymerase chain reaction

Table 2 Visual analog scale (VAS), Sniffin' sticks tests, and short version of questionnaire of olfactory disorders (Short-QOD-NS) results

\begin{tabular}{lrc}
\hline & Mean & SD \\
\hline VAS (subjective \% of olfactory recovery) & 33.9 & 25.6 \\
Sniffin' Sticks test-scores & & \\
Threshold detection & 4.7 & 4.0 \\
Discrimination & 10.3 & 3.1 \\
Identification & 9.4 & 3.9 \\
Short-QOD-NS-total & 11.1 & 5.0 \\
Short-QOD-NS-Eating & 3.1 & 2.2 \\
Short-QOD-NS-Anxiety & 2.1 & 1.0 \\
Short-QOD-NS-Annoyance & 1.0 & 1.1 \\
Short-QOD-NS-Social & 4.7 & 2.5 \\
\hline
\end{tabular}

$S D$ standard deviation

normosmic (TDI $\geq 30.75), 53.7 \%(n=29)$ as hyposmic $(16.25 \leq \mathrm{TDI} \leq 30.5)$, and $22.2 \%(n=12)$ as functional anosmic $(\mathrm{TDI} \leq 16)$. Based on the SST-12, $14.8 \%(n=8)$ of the patients could be classified as normosmic (SST-12 $\geq 11$ ), $48.1 \%(n=26)$ as hyposmic $(6<$ SST $-12<10)$, and $37 \%$ 
Table 3 Categorization of subjects based on the Sniffin' Sticks Test scores (SST), Sniffin' Sticks Test 12 items scores (SST-12), and inter-test reliability (Kappa)

\begin{tabular}{llll}
\hline$N=54$ & Normosmic $N(\%)$ & Hyposmic $N(\%)$ & Anosmic $N(\%)$ \\
\hline SST & $13(24.1)$ & $29(53.7)$ & $12(22.2)$ \\
SST-12 & $8(14.8)$ & $26(48.1)$ & $20(37.0)$ \\
Correct categorization & $4(7.4)$ & $17(31.5)$ & $12(22.2)$ \\
False positive & $0(0)$ & $9(16.7)$ & $8(14.8)$ \\
False negative & $4(7.4)$ & $0(0)$ & $0(0)$ \\
Cohen's Kappa & 0.242 & 0.224 & 0.654 \\
\hline
\end{tabular}

$S D$ standard deviation $(n=20)$ as functional anosmic (SST-12 $\leq 6)$. Interestingly, patients who increased their consummation of salt showed lower SST $(\mathrm{U}=112.5, p=0.003)$ and SST-12 $(\mathrm{U}=121$, $p=0.005)$ scores compared to the patients who did not increase salt usage $(20.0 \pm 8.8$ vs. $27.9 \pm 7.7$, and $5.7 \pm 3.5$ vs. $8.5 \pm 2.2$, respectively). The same result was found for patients who increased their consummation of sugar, that showed lower SST $(U=73.5, p=0.014)$ and SST-12 $(U=62$, $p=0.005)$ scores compared to the other patients $(26.2 \pm 8.0$ vs. $17.1 \pm 8.4$, and $4.6 \pm 2.9$ vs. $6.9 \pm 2.9$, respectively). Interestingly, patients who increased their consummation of sugar had a lower Short-QOD-NS alimentary subscale $1.4 \pm 1.8$ vs. $3.5 \pm 2.0 ; U=66.5, p=0.006$ ). The self-reported presence of taste disorders did not show any significant link with the presence of an increased consummation of salt $\left(\mathrm{Chi}^{2}=0.74\right.$, $p=389)$ or sugar $\left(\mathrm{Chi}^{2}=1.13, p=287\right)$.

Taking SST as the gold standard, on 54 patients, $61 \%$ $(n=33)$ were classified in the same category by the SST12 patients. SST-12 misdiagnosed 4 patients as normosmic (7.4\%), 8 as anosmic (14.8\%), and 9 as hyposmic (16.7\%). Importantly, all the patients who were diagnosed as anosmic by the SST were also detected by the SST-12. Accordingly, Cohen's Kappa coefficient revealed a week agreement between the two tests in classifying patients as normosmic $($ Kappa $=0.24)$ and hyposmic $($ Kappa $=0.22)$, but a strong agreement in classifying patients as anosmic $(\mathrm{Kappa}=0.65)$. The sensitivity and specificity of the SST-12, compared to the SST score, are reported in Table 4 and suggest that a score of 6 is the cut-off that maximizes the combination between specificity (100\%) and sensitivity $(81 \%)$ in detecting anosmic patients. The presence of taste disorders did not affect the type of errors of the SST-12 compared to the SST.

\section{Correlations between self-reported olfactory recovery, SST, SST-12 score, and Short-QOD-NS}

VAS scores were $45 \pm 24 \%$ (range $5-70 \%$ ), $38 \pm 25 \%$ (range $1-90 \%$ ), and $13 \pm 16 \%$ (range $0-50 \%$ ) for, respectively, normosmic, hyposmic, and anosmic patients, based on the SST. Based on the SST-12, VAS scores were $52 \pm 26 \%, 42 \pm 22 \%$, and $16 \pm 19 \%$ for, respectively, normosmic, hyposmic, and anosmic patients. An almost perfect correlation between
Table 4 Sensitivity and specificity of the different cut-off scores of the SST-12 compared to the SST in classifying patients as anosmic

\begin{tabular}{lll}
\hline SST-12 score & Sensitivity & Specificity \\
\hline 0 & 1.000 & 0.00 \\
1 & 1.000 & 0.17 \\
2 & 1.000 & 0.25 \\
3 & 0.98 & 0.33 \\
4 & 0.93 & 0.42 \\
5 & 0.88 & 0.75 \\
$\mathbf{6}$ & $\mathbf{0 . 8 1}$ & $\mathbf{1 . 0 0}$ \\
7 & 0.69 & 1.00 \\
8 & 0.55 & 1.00 \\
9 & 0.38 & 1.00 \\
10 & 0.19 & 1.00 \\
11 & 0.07 & 1.00 \\
12 & 0.00 & 1.00 \\
\hline
\end{tabular}

The cut-off of 6 , which maximizes the sensitivity/specificity ratio, is reported in bold

scores at the SST identification score and SST-12 was found $\left(\mathrm{rho}_{(52)}=0.98, p<0.001\right)$, confirming that the SST-12 can assess odor identification as well as the SST. We observed positive correlation between VAS recovery (\%) and the identification scores for both the SST $\left(\mathrm{rho}_{(52)}=0.47, p<0.001\right)$ and the SST-12 scores $\left(\mathrm{rho}_{(52)}=0.49, p<0.001\right)$, testifying that the two scales were equally correlated to self-reported disorders. These results are reported in Fig. 1.

Olfactory quality of life-We found a significant positive correlation between the SST global score and the global $\left(\mathrm{rho}_{(52)}=0.30, p=0.027\right)$ and social Short-QOD-NS subscales $\left(\mathrm{rho}_{(52)}=0.38, p=0.041\right)$. Concerning identification scores, a significant correlation was found between SST-I and the global Short-QOD-NS $\left(\mathrm{rho}_{(52)}=0.29\right.$, $p=0.036$ ), between SST-I and the Short-QOD-NS eating subscale $\left(\mathrm{rho}_{(52)}=0.29, p=0.036\right)$, between SST-12 and the global Short-QOD-NS $\left(\right.$ rho $\left._{(52)}=0.33 p=0.016\right)$, its eating $\left(\mathrm{rho}_{(52)}=0.32 p=0.017\right)$, and anxiety $\left(\mathrm{rho}_{(52)}=0.29\right.$ $p=0.032$ ) subscales. The only significant correlation with the SST discrimination score was with the anxiety subscale $(\operatorname{rho}(52)=0.27, p=0.048)$. No significant correlation 
Fig. 1 Correlations between a SST-12 and SST identification score, $\mathbf{b}$ percentage of subjective olfactory recovery (VAS) and SST, and $\mathbf{c}$ percentage of subjective olfactory recovery (VAS) and SST-12 a

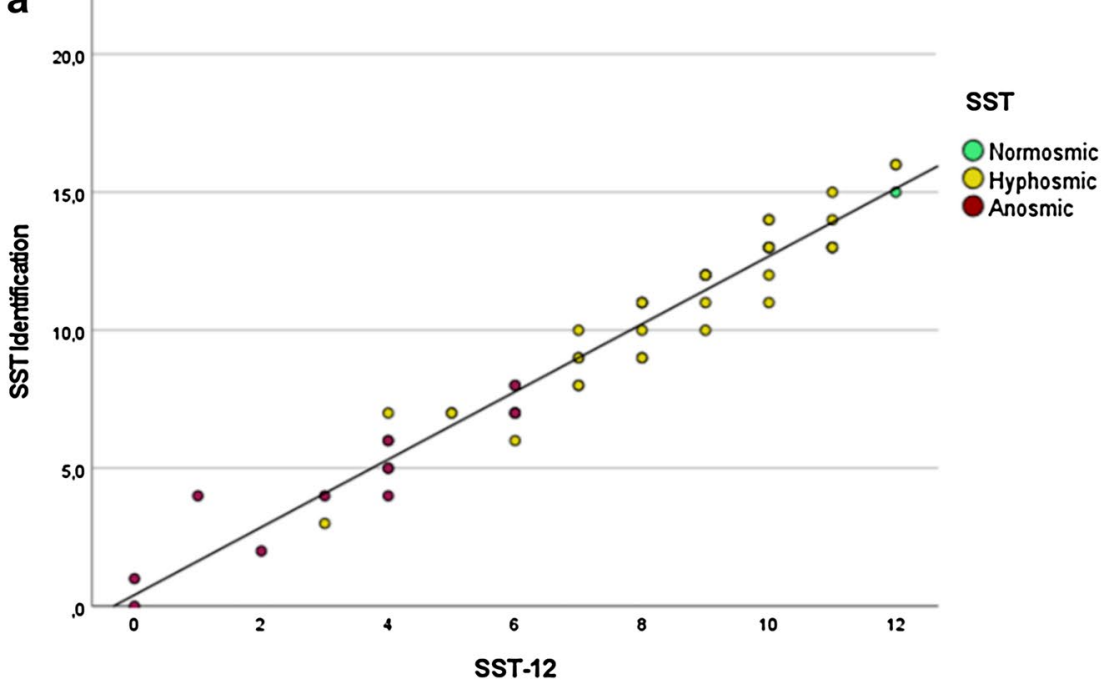

b
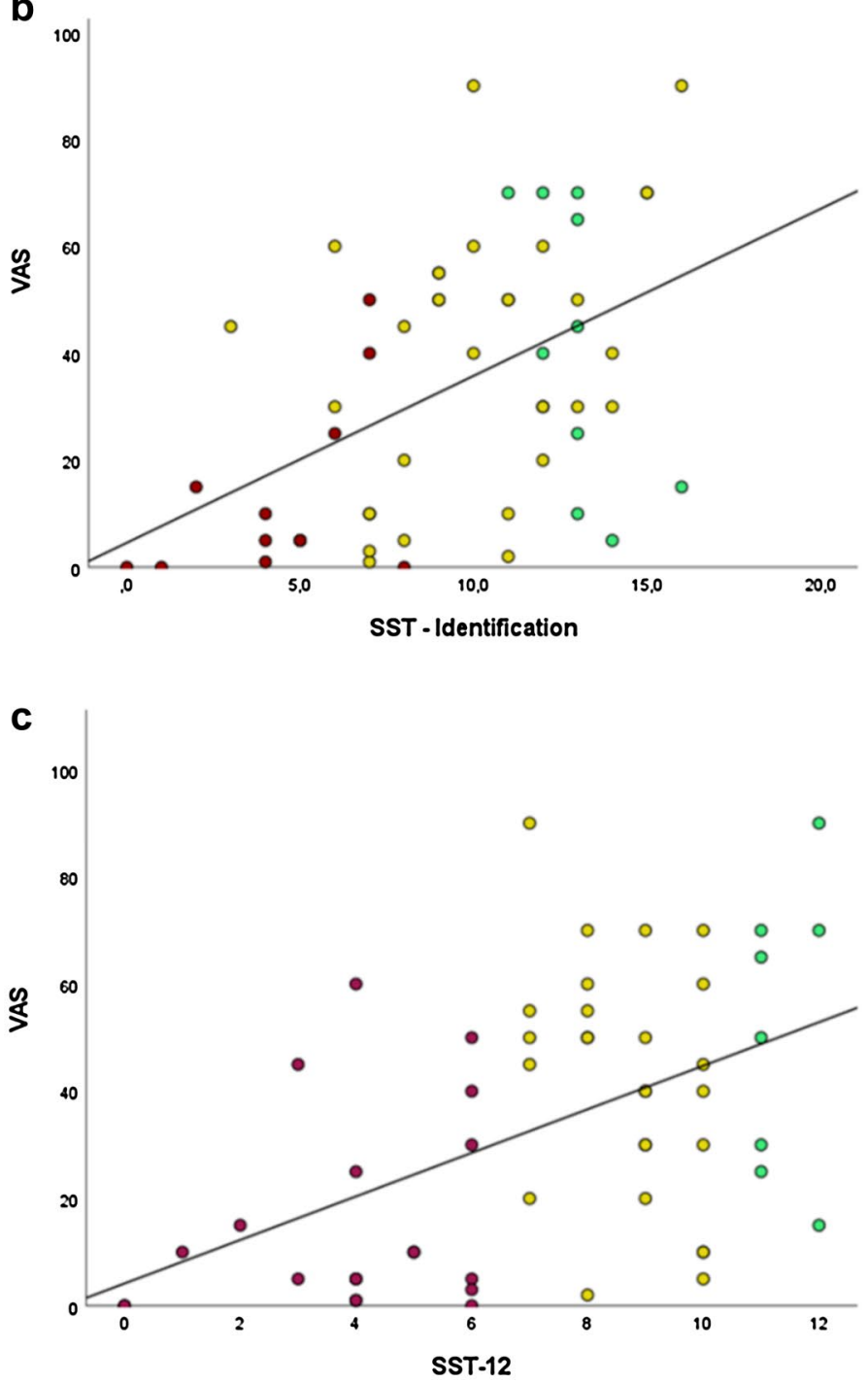

SST

ONormosmic OHyphosmic - Anosmic

SST-12

ONormosmic OHyphosmic - Anosmic 
between SST threshold scale and Short-QOD-NS was found. The VAS score showed positive correlations with the global Short-QOD-NS $\left(\mathrm{rho}_{(52)}=0.39, p=0.003\right)$ and with the Short-QOD-NS social $\left(\mathrm{rho}_{(52)}=0.31, p=0.022\right)$ and eating $\left(\right.$ rho $\left._{(52)}=0.40, p=0.003\right)$ subscales.

\section{Discussion}

This is the first study that evaluates quantitatively the efficiency of the SST-12 to screen for post-COVID-19 olfactory disorders, and specially to identify post-COVID-19 anosmic patients.

Screening for olfactory disorders is important because, in addition to allowing to set up appropriate care for patients, it helps prevent the occurrence of consequences of long-term anosmia, like an alteration in the quality of life [2], bad diet habits [3], changes in social relations [4], psychiatric disorders, such as depression [6], anxiety, or anorexia [7], its nutritional consequences [8], and finally cognitive impairment [4]. Although a subjective olfactory complaint $(80 \%$ anosmia, 20\% hyposmia) is now a very frequent symptom of a COVID-19 infection [32] affecting 70 to $85 \%$ of patients $[33,34]$, only $21 \%$ of clinicians use psychophysical olfactory tests to characterize this olfactory complaint [35].

The present study shows the reliability of SST-12 in screening for post-COVID-19 olfactory disorders, and in particular anosmia. Among diagnostic errors, only 7\% $(n=4)$ of hyposmic patients would have been considered normosmic by SST-12. The other differences in scores between the SST-12 and the SST do not modify the purpose of the screening, which is to perform or have performed complete olfactory tests in the event of an abnormality detected. In this way, all but 4 patients (92\%) would have been correctly screened using the SST-12. All the postCOVID-19 anosmic patients at the SST were correctly screened by the SST-12 as evidenced by the "good" correlation coefficient (0.61 $\leq$ Kappa $\leq 0,80$-Altman 1991). Full identification subdomain test of SST, with 16 items, is often used in COVID-19 olfactory complain assessment $[34,36,37]$ but, although already validated [38], remains longer, and uses garlic, turpentine, apple, and anis which are poorly identified by normosmic patients and raising the risk of overestimating the olfactory impairment [19]. SST12 thus seems to be perfectly suited to face the challenge of large cohort quick (four minutes [19]) screening potentially impaired with olfactory disorder, especially concerning general practitioners.

The total SST-12, as SST, was significantly correlated $(p<0.001)$ with odor complaint (VAS-Fig. 1) which reflects persistent post-COVID-19 olfactory impairment, i.e., mainly identification disorders rather than threshold or discrimination [2] as we published previously. Post-COVID-19 olfactory disorders show unique psychophysical characteristics consistent with a central olfactory impairment $[2,15]$. In a population of 34 patients deprived of their olfaction for about 6 months after COVID-19 and presenting a persistent odor complaint (VAS), we previously highlighted a significantly predominant, and gradually worsening, odor identification impairment [2], as Ianuzzi et al. [20] underlined a lack of identification recovery in their 30 patients study. Unlike the SST, the SST-12 only assesses the identification of odors and thus seems more suited to detect an identification disorder than the SST, which adds to the identification score, a score for discrimination, and perception of the odor threshold. As shown in previous post-COVID-19 olfactory impairment studies [2,36], the SST interpretation can conclude to a global normosmia even if patients actively complain about their olfaction. The full SST (i.e., threshold, discrimination, and identification global score) might therefore not be used as a gold standard in the post-COVID-19 odor evaluation given that some patients, early [37] and at a distance [2] from SARS-COV-2 infection, may be incorrectly classified as normosmic on SST despite olfactory complain [2].

In this study we found that olfactory complain (VAS) was significantly linked to an SST or SST12 impairment, justifying the no need to a psychophysical screening test to take social distancing and barrier measures in case of acute olfactory disorders in COVID-19 pandemic times. Moreover, $7 \%$ of hyposmic patients could be missed with such a test. This is especially true since such a screening test poses a contamination risk to the examiner. However, at a distance from acute infection, SST-12 could be helpful to screen post-COVID-19 olfactory disorders. In case of a complaining person, as our results suggest, a complete psychophysical olfactory test might be directly performed as an olfactory complain is highly correlated with an impaired SST and SST-12. But in case of a non-complaining, or olfactory impairment unaware, post-COVID-19 patient, SST-12 could prevent negative consequences of unknown olfactory disorders, especially quality of life [39] and metabolic impairments.

Indeed, smell loss causes a significant deterioration in the quality of life [39]. QOD and specifically QODNS [26, 39] are often used for ENT olfaction assessment but could be a problem in clinical research as patients' mental burden could be important. The Mattos et al. [28] short version has been chosen in this work as it shows a strong correlation with QOD-NS total and subdomainspecific scores. Less time consuming, it better fits with the constraints of the routine clinical assessment. In our study, Short-QOD-NS results underlined the quality of life impairment of an olfactory loss and show a significant correlation with SST, SST-12, and VAS results, specifically when identification is impaired, as we published in a previous work [2]. Short-QOD-NS subscales analysis 
showed mainly that eating subscale is always significantly impaired. This could explain that 45.5 and $20.5 \%(n=44)$ of post-COVID-19 patients increased, respectively, their daily diet salt and sugar intake. As previously published, salt and sugar intake increased concerned near $30 \%$ of COVID-19 patients [40], especially young women. Our results suggest that these bad diet habits could concern in fact olfactory impaired post-COVID-19 patients, especially anosmic ones (SST-12 $\leq 6)$ being deprived of their original food tastes and trying to enhance it whatever the way. Interestingly, there is no significant relation, otherwise only with the SST-12 score, between the risk of bad diet habits and subjective olfactory complain, underlining the benefits of using SST-12. The sugar intake is also concerned as COVID-19 could basically raise blood glucose and HbA1c levels [41], which has to be monitored after hospital discharge. It is a major public health concern as post-COVID-19 olfaction disorders recovery time is still uncertain and long-term salt and sugar intake could increase, respectively, blood pressure [42] and type 2 diabetes [43] onset and so cardiovascular risk. In case of SST-12 screened anosmia, a not to change daily use of salt and sugar advice must be added to the patient consultation.

Despite these interesting results, this study suffers from some limitations. The main limitation is our cohort size (54 patients), with no follow-up reported, who spontaneously consulted our university hospital, which represents the risk of a recruitment bias. The small sample size and the high number of subjective variables in this study (VAS, SST, SST-12, sugar and salt consumption) may have contributed to a limited strength of correlations (rho(32) MAX $=0.49$ ), and therefore, our results cannot be directly generalized to all patients with a post-COVID olfactory disorder and should be verified in a larger prospective cohort study. Patients recruitment at different times from their post-COVID-19 olfactory loss introduces heterogeneity in the analysis, as many cases recover over time [44], but allowed us to evaluate SST-12 to many different impaired patients. Even if it could be a bias, we have chosen not to exclude patients who took corticosteroids because of weak evidences of its usefulness on olfactory recovery after a post-viral olfactory loss, especially in COVID-19 [45].

\section{Conclusion}

The SST-12 is an olfactory psychophysical test suitable for quick screening an olfactory sequelae post-COVID-19. Its use in the context of screening for a long olfactory COVID could be used for the implementation of personalized general practice management of olfactory disorders and the prevention of psychological and metabolic consequences.

\section{Declarations}

Competing interests The authors declare no competing interests.

\section{References}

1. Gerkin RC, Ohla K, Veldhuizen MG et al (2020) Recent smell loss is the best predictor of COVID-19 among individuals with recent respiratory symptoms. Chem Senses 2021(46):1-12

2. Vandersteen C, Payne M, Dumas L-E et al (2021) Persistent olfactory complaints after COVID-19: a new interpretation of the psychophysical olfactory scores. Rhinol Online 4(14):66-72

3. Aschenbrenner K, Hummel C, Teszmer K et al (2008) The influence of olfactory loss on dietary behaviors. Laryngoscope 118(1):135-144

4. Valsamidis K, Printza A, Constantinidis J, Triaridis S (2020) The impact of olfactory dysfunction on the psychological status and quality of life of patients with nasal obstruction and septal deviation. Int Arch Otorhinolaryngol 24(02):e237-e246

5. Schablitzky S, Pause BM (2014) Sadness might isolate you in a non-smelling world: olfactory perception and depression. Front Psychol 5:2

6. Hur K, Choi JS, Zheng M, Shen J, Wrobel B (2018) Association of alterations in smell and taste with depression in older adults. Laryngoscope Investig Otolaryngol 3(2):94-99

7. Croy I, Nordin S, Hummel T (2014) Olfactory disorders and quality of life-an updated review. Chem Senses 39(3):185-194

8. Nordin S (2009) Sensory perception of food and ageing. Food for the ageing population. Elsevier, pp 73-94

9. Boscolo-Rizzo P, Guida F, Polesel J et al (2021) Self-reported smell and taste recovery in coronavirus disease 2019 patients: a one-year prospective study. Eur Arch Oto-Rhino-Laryngol 2:0123456789

10. Doty RL (2007) Office procedures for quantitative assessment of olfactory function. Am J Rhinol 21(4):460-473

11. Su B, Bleier B, Wei Y, Wu D (2021) Clinical implications of psychophysical olfactory testing: assessment, diagnosis, and treatment outcome. Front Neurosci 15:1-12

12. Hummel T, Whitcroft KL, Andrews $P$ et al (2017) Position paper on olfactory dysfunction. Rhinol J 54(26):1-30

13. Oleszkiewicz A, Schriever VA, Croy I, Hähner A, Hummel T (2019) Updated Sniffin' Sticks normative data based on an extended sample of 9139 subjects. Eur Arch Oto-Rhino-Laryngology 276(3):719-728

14. Sorokowska A, Schriever VA, Gudziol V et al (2015) Changes of olfactory abilities in relation to age: odor identification in more than 1400 people aged 4 to 80 years. Eur Arch Oto-Rhino-Laryngology 272(8):1937-1944

15. Whitcroft KL, Cuevas M, Haehner A, Hummel T (2017) Patterns of olfactory impairment reflect underlying disease etiology. Laryngoscope 127(2):291-295

16. Sorokowska A, Oleszkiewicz A, Minovi A, Konnerth CG, Hummel T (2019) Fast screening of olfactory function using the Q-sticks test. ORL 81(5-6):245-251

17. Doty RL, Marcus A, William LW (1996) Development of the 12-item cross-cultural smell identification test(CC-SIT). Laryngoscope 106(3):353-356 
18. Jackman AH, Doty RL (2005) Utility of a three-item smell identification test in detecting olfactory dysfunction. Laryngoscope 115(12):2209-2212

19. Hummel T, Rosenheim K, Konnerth C-G, Kobal G (2001) Screening of olfactory function with a four-minute odor identification test: reliability, normative data, and investigations in patients with olfactory loss. Ann Otol Rhinol Laryngol 110(10):976-981

20. Iannuzzi L, Salzo AE, Angarano G et al (2020) Gaining back what is lost: recovering the sense of smell in mild to moderate patients after COVID-19. Chem Senses 45(9):875-881

21. Hummel T, Sekinger B, Wolf SR, Pauli E, Kobal G (1997) 'Sniffin' sticks': olfactory performance assessed by the combined testing of odour identification, odor discrimination and olfactory threshold. Chem Senses 22(1):39-52

22. Allis TJ, Leopold DA (2012) Smell and taste disorders. Facial Plast Surg Clin North Am 20(1):93-111

23. Rumeau C, Nguyen DT, Jankowski R (2016) Comment tester l'olfaction avec le Sniffin' Sticks test ${ }^{\circledR}$. Ann françaises d'Otorhino-laryngologie Pathol Cervico-faciale 133(3):183-186

24. Brämerson A, Nordin S, Bende M (2007) Clinical experience with patients with olfactory complaints, and their quality of life. Acta Otolaryngol 127(2):167-174

25. Frasnelli J, Hummel T (2005) Olfactory dysfunction and daily life. Eur Arch Oto-Rhino-Laryngology 262(3):231-235

26. Mattos JL, Schlosser RJ, DeConde AS et al (2018) Factor analysis of the questionnaire of olfactory disorders in patients with chronic rhinosinusitis. Int Forum Allergy Rhinol 8(7):777-782

27. Simopoulos E, Katotomichelakis M, Gouveris H, Tripsianis G, Livaditis M, Danielides V (2012) Olfaction-associated quality of life in chronic rhinosinusitis: adaptation and validation of an olfaction-specific questionnaire. Laryngoscope 122(7):1450-1454

28. Mattos JL, Edwards C, Schlosser RJ et al (2019) A brief version of the questionnaire of olfactory disorders in patients with chronic rhinosinusitis. Int Forum Allergy Rhinol 9(10):1144-1150

29. Smeets MAM, Veldhuizen MG, Galle $S$ et al (2009) Sense of smell disorder and health-related quality of life. Rehabil Psychol 54(4):404-412

30. Han P, Su T, Qin M, Chen H, Hummel T (2020) A systematic review of olfactory related questionnaires and scales. Rhinol J. 59(2):2

31. Leclercq C, Chiesa-Estomba CM, Horoi M et al (2021) Validity and reliability of the french short version of the questionnaire of olfactory disorders-negative statements (sQOD-NS). Ear, Nose Throat J 2:2

32. Gane SB, Kelly C, Hopkins C (2020) Isolated sudden onset anosmia in COVID-19 infection. A novel syndrome? Rhinol J. 58(3):299-301

33. Lechien JR, Chiesa-Estomba CM, Place $S$ et al (2020) Clinical and epidemiological characteristics of 1420 European patients with mild-to-moderate coronavirus disease 2019. J Intern Med 288(3):335-344
34. Lechien JR, Cabaraux P, Chiesa-Estomba CM et al (2020) Objective olfactory evaluation of self-reported loss of smell in a case series of 86 COVID-19 patients. Head Neck 42(7):1583-1590

35. Agyeman AA, Chin KL, Landersdorfer CB, Liew D, OforiAsenso R (2020) Smell and taste dysfunction in patients with COVID-19: a systematic review and meta-analysis. Mayo Clin Proc 95(8):1621-1631

36. Le Bon SD, Pisarski N, Verbeke J et al (2021) Psychophysical evaluation of chemosensory functions 5 weeks after olfactory loss due to COVID-19: a prospective cohort study on 72 patients. Eur Arch Oto-Rhino-Laryngology 278(1):101-108

37. Lechien JR, Cabaraux P, Chiesa-Estomba CM et al (2020) Psychophysical olfactory tests and detection of COVID-19 in patients with sudden onset olfactory dysfunction: a prospective study. Ear, Nose Throat J 99(9):579-583

38. Mueller CA, Grassinger E, Naka A, Temmel AFP, Hummel T, Kobal G (2006) A self-administered odor identification test procedure using the 'Sniffin', Sticks"'. Chem Senses 31(6):595-598

39. Shu CH, Lee PO, Lan MY, Lee YL (2011) Factors affecting the impact of olfactory loss on the quality of life and emotional coping ability. Rhinol J 49(3):337-341

40. Rolland B, Haesebaert F, Zante E, Benyamina A, Haesebaert J, Franck N (2020) Global changes and factors of increase in caloric/ salty food intake, screen use, and substance use during the early COVID-19 containment phase in the general population in France: survey study. JMIR Public Heal Surveill. 6(3):e19630

41. Chen J, Wu C, Wang X, Yu J, Sun Z (2020) The impact of COVID-19 on blood glucose: a systematic review and meta-analysis. Front Endocrinol (Lausanne) 11(October):1-8

42. Graudal NA, Hubeck-Graudal T, Jurgens G (2017) Effects of low sodium diet versus high sodium diet on blood pressure, renin, aldosterone, catecholamines, cholesterol, and triglyceride. Cochrane Database Syst Rev 2017:4

43. Lean MEJ, Te Morenga L (2016) Sugar and type 2 diabetes. $\mathrm{Br}$ Med Bull 120(1):43-53

44. Hopkins C, Surda P, Vaira LA et al (2020) Six month follow-up of self-reported loss of smell during the COVID-19 pandemic. Rhinol J. 11:2

45. Huart C, Philpott CM, Altundag A et al (2021) Systemic corticosteroids in coronavirus disease 2019 (COVID-19)-related smell dysfunction: an international view. Int Forum Allergy Rhinol 11(7):1041-1046

Publisher's Note Springer Nature remains neutral with regard to jurisdictional claims in published maps and institutional affiliations. 